\title{
An Efficient way of Predicting Covid-19 using Machine and Deep Learning Algorithms
}

\author{
Meenakshi $\mathrm{N}^{1}$, Kumaresan Angappan ${ }^{2}$,Sandhya \\ $\mathrm{A}^{3}$,NagaSusmitha $\mathrm{V}^{4}$,Vaishnavi $\mathrm{K}^{5}$ \\ \{abu221985@gmail.com¹, kummaresan@gmail.com², \\ chenchusandhya1999@gmail.com ${ }^{3}$, \\ susmithasubramanyam1234@gmail.com ${ }^{4}$,vaishnavi12345@gmail.com ${ }^{5}$ \} \\ Department of Information Technology, Hindustan Institute \\ of Technology and Science, Chennai, India ${ }^{1,2,3,4}$
}

\begin{abstract}
Recently the novel coronavirus disease pushed the world into the dramatic situation. The tough thing to deal with novel corona virus is the prediction. In the beginning RT PCR test is the golden standard test for the prediction of COVID, which takes more time, more licensed laboratories, trained personnel and prediction accuracy will be not fruitful. In our System, We used current technology for the prediction, which involves: An Efficient Random Forest, a machine learning classification model which predicts whether the person is Corona affected or not using routine blood reports and a deep learning model, Modified DenseNet121 which was pre-trained to predict theCovid using CT scan images. To analyze the machine learning model performance, 5744 blood report samples have been collected from Kagglerepository;similarly, 2482 CT scan samples have been collected from the Kaggle repository, for prediction using Random Forest and DenseNet121 model. The proposed model which is developed using machine and deep learning techniques can be deployed easily and can be used for rapid and accurate prediction of Covid19.
\end{abstract}

Keywords- COVID-19, clinical data analysis, Machine Learning, Deep Learning, Blood test, CT scan, Efficient Random Forest, Modified DenseNet121.

\section{Introduction}

COVID 19 [1][2] is an infection which occurs atrespiratory organs caused by SARSCoV-2 virus. This novel coronavirusseverely affects the acute respiratory syndrome coronavirus 2 which is first appeared in Wuhan, China in December 2019.On 11th March,2020 WorldHealth Organization(WHO) announced upsurgeof Covid19 as pandemic in the global level. The growth of Coronavirus will be more when people come contact with other people directly. So, there it was mandatory to stop people travelling from one location to the other. And it is advised to wash the hands repeatedly for every 1 hour. This step may not prevent Corona however make us safe from being Corona affected. The general 
symptoms which was exhibited in many people are cough and fever and other symptoms may occur, including chest discomfort, sputum development.

The Coronavirus disease first started as an infection in throat along with any of the following symptoms : Cough, fever, body pains, headache, loss of smell and taste. It will easily spreads from one person to another person when they make direct contact with covid19 infected patients. Jiang and his team [3] conducted a research and found $4.5 \%$ as death rate with Covid19 infection across the world. Covid positive patients with age range of 70 to 79 years the death rate is $8.0 \%$, and for patients above 80 years the death rate is $14.8 \%$. It is also confirmed that the risk prediction of Corona Virus is at peek zone for the patients who are above the age of 50 years with chronic illnesses therefore they should take special precautions, care and attention. One of the pressing issue with Covid 19 is rapid spread of the disease that means if around 20 people are affected with COVID-19 positive, then they are more likely to infect $25-45$ other people [4] within their community. Therefore, COVID 19 can be affected to large group of people with in less span of time until or unless some preventive measures are implemented.

At the beginning of the pandemic, RT-PCR [5], a gold standard test which is used to detect COVID-19 infection. Real-time Reverse Transcription Polymerase Chain Reaction (RT-PCR), generally this test analysis the De-oxyribo Nucleic Acids (DNA), however DNA is not collected directly, RNA is collected and then the collected RNA has to be converted to DNA because of this it is "Reverse Transcription". In this test first swab test is performed to collect nasalsecrations and then the secrataions are analysed to find the Corona. But, lack of equipment and high standard Isolated laboratories for screening limited the fast and accurate testing. Later, the reverse transcription polymerase chain reaction (RT-PCR) [5] also giving false results.

In Wuhan China, clinical study with 1015 patients has shown that chest computed tomography(CT) analysis can achieve 0.97 of sensitivity, 0.68 of accuracy , 0.25 of specificity to detect COVID 19 disease, with RT-PCR [6]. Symptoms of coronavirus in lower parts of lungs gives more accuracy by using CT Scan[7][8][9] than using RT-PCR. To assist radiologists for the prediction of COVID 19 using CT Scan, we need to improve the system speed(prediction of COVID 19). This can be done by using Automatic diagnostic system, uses Machine Learning (changes in blood parameters of COVID-19 patients plays an important role in predicting coronavirus infection) and Deep Learning algorithms. Even for an experienced physicianit is difficult to extract all the necessary information from blood tests. Machine Learning algorithms can learn and distinguish various patterns from blood report analysis. Blood tests based ML System approach for Covid detection provides easy to use, a fast, accessible less expensive and more accessible less alternative to costly and time taking methods(like imaging based method and RT-PCR test. This approach is to decrease the mortality rate, the time, spread ratio and efforts by narrow down the testing time and produce fast and accurate results.

In our Model we are using :

- Machine Learning and Deep Learning algorithms[7] based diagnostic system used for early detection and fast assessment of risk detection in Coronavirus infection and it can be easily deployed.

- The ML Algorithms we are used : Random Forest[10], Decision Tree[11], Logistic Regression, Support Vector Machine[12] to detect negative/positive results of COVID-19 patients from blood reports and found Random Forest as best algorithm

- And DL Algorithm Modified DENSENET 121 is used for diagnosis of COVID-19 infection through CT Scan images. 
- Integration of blood reports( laboratory/clinical blood reports) and radiological data (CT Scan) used to detect the Coronavirus presence.

In this Model, we concluded that Machine Learning and Deep Learning based classifier provides a diagnostic tool for prediction of COVID-19 using blood report samples, and CT Scan images.

\section{RELATED WORKS}

Beginning from the flare-up of the Covid numerous specialists are dealing with this, finding a wide range of ways for recognizing Coronavirus and just as for its cure. It is hard to distinguish uncovered people since they don't show infection indications right away. Along these lines, it is important to discover a strategy for assessing the quantity of conceivably contaminated people consistently to receive the suitable measures. Machine Learning and Deep Learning can be utilized to inspect an individual for Coronavirus as an option in contrast to customary techniques which are tedious and expensive. Although there are a few investigations on Coronavirus, this examination zeroed in on the utilization of ML in diagnosing patients for Coronavirus disease through Blood Reports, and CT Scan pictures.

Many researches have been done in this area(Machine Learning) and found the greatest advantage of Machine Learning, which can be trained with the existing data, further we can use that trained model for the prediction of the Covid 19. In our Model, Machine Learning was implemented by considering blood report samples to predict whether a patient is positive for COVID-19 and used Deep Learning based Densenet technique by using CT Scan pictures. 2.1COVID-19 DIAGNOSIS USING DEEP LEARNING MODEL :

It has been observed that the usage of Artificial Intelligence, Machine and Deep Learning has been increasing day by day in various field (example: medicine ,malware detection )[13],[14]. In 2012, deep learning a modern Machine learning system was introduced, which is based on Densely connected convolutional network a model of Deep Learning[20]. Deep learning consists of different techniques to deal with various problems, it comprises various layers for learning the data hidden in the images and from that learning it takes some decisions which we can use those decisions for the prediction. According to Le Cun and his team members[15], deep-learning techniques provides more accuracies and can speedup the output when compared with humans

\subsubsection{CT Scan Diagnosis Using Deep Learning Model :}

Godfrey Hounsfield and Allan Cormack developed CT Scan 1972. The main advantage of CT scanning does not involve any pain, helps for precise prediction, consists of threedimensional images [16 [17].

Jiang and his team[18] compared RT-PCR and CT Scans for 51 patients which comprises 29 men and 22 women with the history of travel to or residency in pandemic areas and founded that they are exhibiting the symptoms of fever and their CT Scan reports showed that they are having respiratory infection. They found sensitivity of the CT Scan reports as $98 \%$, while the sensitivity of the golden standard RT-PCR found to be $71 \%$.

Wang and his team [19] developed a deep-learning approach to extract the information( to understand the Covid19 features) from CT scans. Their study included a collection of 453 CT scans from 99 patients. They extracted 195 regions of interest (ROIs) of sizes ranging 
from $395 \times 223$ to $636 \times 533$ pixels from the CT scans of 44 COVID-19-positive pneumonia patients and 258 ROIs from those of 50 COVID-19-negative patients. They have applied a modified network inception model and gained an accuracy of $82.9 \%$ for the internal validation with a specificity of around $80.5 \%$ and sensitivity of the model stand up at $84 \%$. They tested the model with external dataset and that testing showed results as $73.1 \%$ as total accuracy and specificity and sensitivity of the model is $67 \%$ and $74 \%$ respectively.

\subsection{COVID-19 \\ INFECTION \\ PREDICTION \\ USING \\ MACHINE}

\section{LEARNINGTECHNIQUES:}

Machine Learning is one of the largest technology in the Artificial Intelligence which comprises Deep Learning. It uses the concepts of mathematics like regression, probability etc to learn and analyze the interesting patterns from the data. Machine learning models are applied to a system and patterns are recongnised, the obtained validation data are then divided according to the patterns learned during the learning or training process. Hence machine learning can be used to predict the behavior from blood reports to decrease the spreading of virus in the early stages.

Wu along with his team members made the first study with the help of Random Forest Algorithm, a classification model of Machine Learning algorithm to detect covid 19 from samples of blood reports. They collected dataset which comprises of 253 blood report data of which 105 blood report data consists of COVID-19 cases, the data is collected from different hospitals in Lanzhou, China. From the sample blood report they identified 11 features as key features out of 49 features which they examined. Some of the key features are Leukocytes, Monocytes, Red Blood Cells etc.

Then the Random Forest model is built and then trained with 11 key features and resulted are noted. In results we can observe that model showed sensitivity of $95.12 \%$, and specificity around $96.97 \%$ comprising the overall accuracy of the model as $96.95 \%$.

However the performance of the model with the external data is not compromising, it was bit lower compared with trained result.

In addition to that the dataset is biased towards covid 19 positive data therefore the performance of the model will suffer for non Covid patient sample blood report $\mathrm{s}$ in the dataset.

\section{An Efficient Random Forest Algorithm and Modified Densenet-121}

\section{Architecture}

In our Model, we are using two algorithms. Machine Learning Algorithm(An Efficient Random Forest) was implemented by considering blood report samples and Deep Learning based Densenet technique implemented by using CT Scan picturesto predict whether a patient is positive for COVID-19 or not.

\subsection{Efficient Random Forest Algorithm:}

Random Forest is one of the machine learning model which is used for classification. It is supervised learning model which means the model is trained with the training data and is tested with new sample of data (test data). 
It is used for classification purpose and regression purpose and it is an ensemble learning model, it supports hyper parameter tuning all these together make the model more efficient.As the name suggests it considers multiple decision tree output to produce the final output, this is the advantage of the efficient Random Forest algorithm, it considers the output with majority votes by not depending on the single tree output. Thus increases the accuracy of the Random Forest Algorithm. The greater number of trees in theforest leads to higher accuracy and prevents the problem of overfitting byaveraging the result . One more advantage with Random Forest is, itsupports Hyper Parameter tuning whichagain increases thePrediction accuracy.

Efficient Random Forest Algorithm consists of two steps,

Creation of Random Forest

ii) Predicting from the created Random Forest

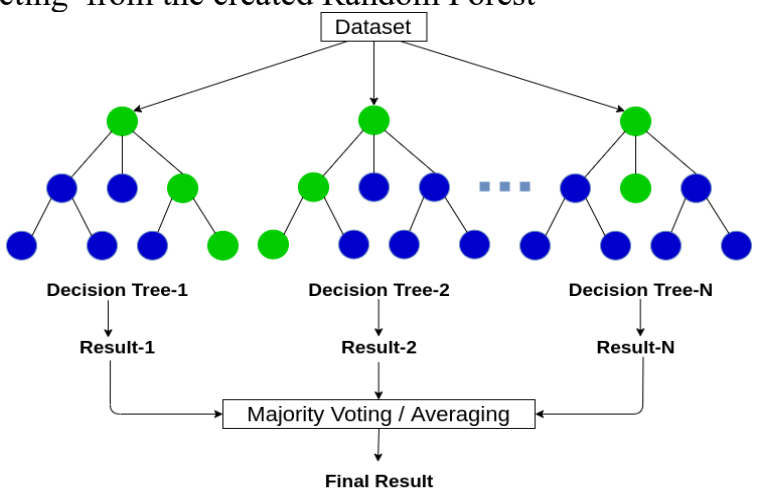

Fig. (1) Random Forest Model

The table shows the list of 21 attributes that are significant for the experiment.

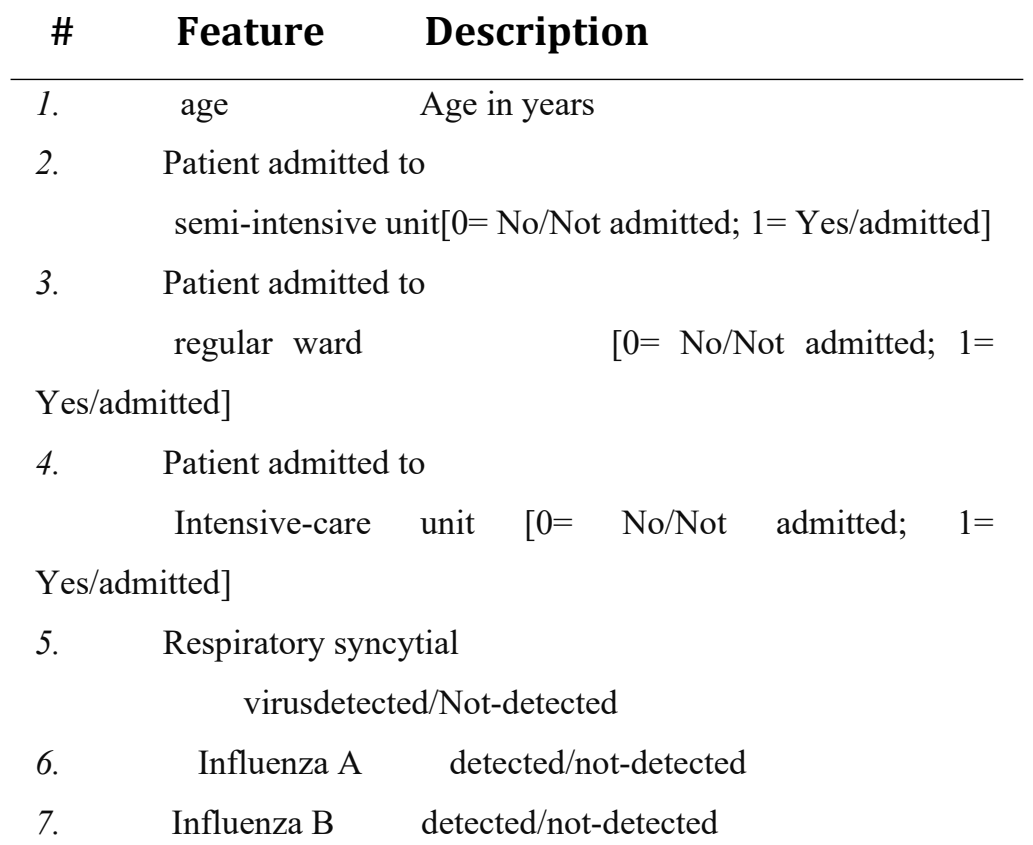




$\begin{array}{ccc}\text { 8. } & \text { Parainfluenza } 1 & \text { detected/not-detected } \\ 9 . & \text { coronavirusNL63detected/not-detected } \\ \text { 10. } & \text { Rhinovirus/Enterovirusdetected/not-detected } \\ \text { 11. } & \text { Coronavirus HKU1 } & \text { detected/not-detected } \\ 12 . & \text { Parainfluenza } 2 & \text { detected/not-detected } \\ 13 . & \text { Parainfluenza } 3 & \text { detected/not-detected } \\ 14 . & \text { Adenovirus } & \text { detected/not-detected } \\ 15 . & \text { Parainfluenza } 4 & \text { detected/not-detected } \\ 16 . & \text { Coronavirus } 229 \mathrm{E} & \text { detected/not-detected } \\ 17 . & \text { CoronavirusOC43 } & \text { detected/not-detected } \\ 18 . & \text { Inf A H1N1 2009 } & \text { detected/not-detected } \\ 19 . & \text { Metapneumovirusdetected/not-detected } \\ 20 . & \text { Bordetella pertussis } & \text { detected/not-detected } \\ 21 . & \text { Chlamydophiladetected/not-detected }\end{array}$

Table. (1) List of attributes

In the Hyper Parameter Tuning (used to inform the model how fastly it has to learn, it is like controlling the behavior of the model) we used number of estimators as " 600 " and criteria to be followed as "Gini Index", minimum number of splits is 5, and minimum sample leaves is 1 , and maximum features has to be learned is auto and random_state to be 1 .

- We used dataset consisting of 5644 data

- We considered more number of attributes which enables the model to predict accuracy.

\subsection{MODIFIEDDENSE NET ARCHITECTURE (DENSENET 121):}

Densenet(121) is the Deep Learning Architecture (transfer model) which is pertained on huge Image dataset. It is especially designed for Image Classification and Prediction. Modified Denset 121 is a part of Convolutional Neural Network, in this DenseNet the layers are connected to each other through Dense Blocks. Each layer in the architecture produces the output in which this output is considered as input for the succeeding layers. Dense net consists of layers as a block (Dense Block), where layers are densely connected to each other, so this makes the algorithm to learn the features fastly and accurately.

The main components in theModified DenseNet architecture are:

1.Dense Blocks and 2.Transition Layers.

1. DenseBlock : It consists of multiple convolution blocks (layers ) each one having the equal number of output channels, but, in the channel dimension we combines both input and output channels.

2. Transition Layers : If we increase the dense block then the number of channels will also be increasing which in turn makes the model complex. To reduce them we use Transition layers . So basically it is used to control the model complexity along with that it reduces the channel number. To achieve this ot uses the concept of $1 * 1$ convolution layer. 


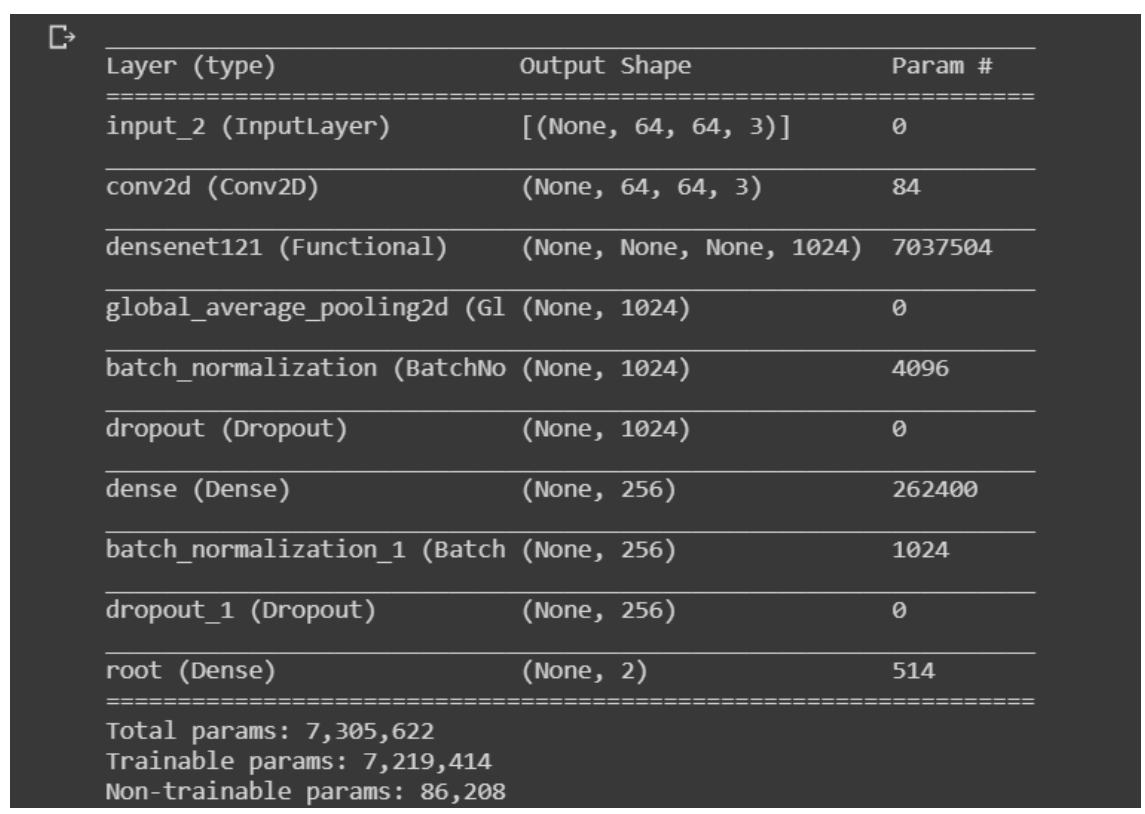

Fig. (2) Modified Densenet-121 Model

To predict Covid19 usingCTSCan our denset121 architecture consists of following layers:

Input Layer : Image height, image width and number of input channels (red, green ,blue are considered for our image which is 3 ). We used image size as $64 * 64$.

ii. Conv 2d (convolutional 2D): We are using CT Scan because the effectiveness of prediction of Covid19 will be great and in general to predict presence of Covid19 using CT Scan requires doctors keen observation and sometimes doctors prediction might go wrong. Here the technology comes into picture.With the help of Deep Learning Convolution NeuralNetworks(DenseNet121) we can reduce the task for the doctor and prediction will be accurate as we are training more dataset and tested to guarantee the accuracy of the model.

iii.GlobalAverage_Pooling:In transition layers we are reducing number of channels to half of the excisting channels. Here, there are $1 * 1$ conv layer and $2 * 2$ average pooling layer with stride of size 2.At last, we have globalAveragepooling, followed by final output layer.GlobalAveragePooling is applied on denseblock to make the connections.

\section{METHODOLOGY :}

\section{1 . Dataset:}

The dataset which was used to train the model is to predict COVID-19 and was gathered from Kagglerepository.It contains 5644 data about COVID-19 infected patients with 111 attributes, but all the the experiments refer to use a subset of 21 attributes. The attributes are 
categorical, real and integer values. The dataset classify into two i.e., training and testing dataset.

And also we collected a datasets containingCT scan images to predict covid-19 from Kaggle Repository which contains 2 classes I.eCovid, Non-Covid.Because of limited availability of ct scan images we applied dataaugumentation.Data Augumentation methods are used commonly in Machine Learning to address class Imbalance problems,overfitting can be reduced in deep learning and improves convergence, which ultimately gives better results.

\subsection{Data analysis}

Data analysis performs major tasks such as data cleaning and integration, identification of missing values and removing the redundant data. Pandas recognizes all the features as numerical either as integers or floats. But some of the attributes are categorical in the dataset as their numbers represent mappings to a real-world phenomenon. Hence, Exploratory Data analysis is performed where first, the list of all the features which are numerical is saved in a column in order to not mix in the analysis with categorical variables. Using Pandas Profiling, distribution of each variable is visualized and a detailed profile report is generated with inference type, quantile statistics, descriptive statistics etc. for exploratory data analysis.

The interactions between each variable is defined with their correlations such as Pearson's correlation coefficient for the measure of linear correlation between two variables; Spearman's correlation coefficient for detecting the nonlinear monotonic correlations between the variables and Cramer's V coefficient for association measure of nominal random variables.

To pick the features that have best relationship with the performance variable, statistical tests are used. The SelectKBest class from the scikit-learn library is used to select features of different statistical tests. The proposed system uses the Chi-Squared test for non-negative features to select best features from the dataset and then ranks the features according to their importance as shown in Fig. (3).

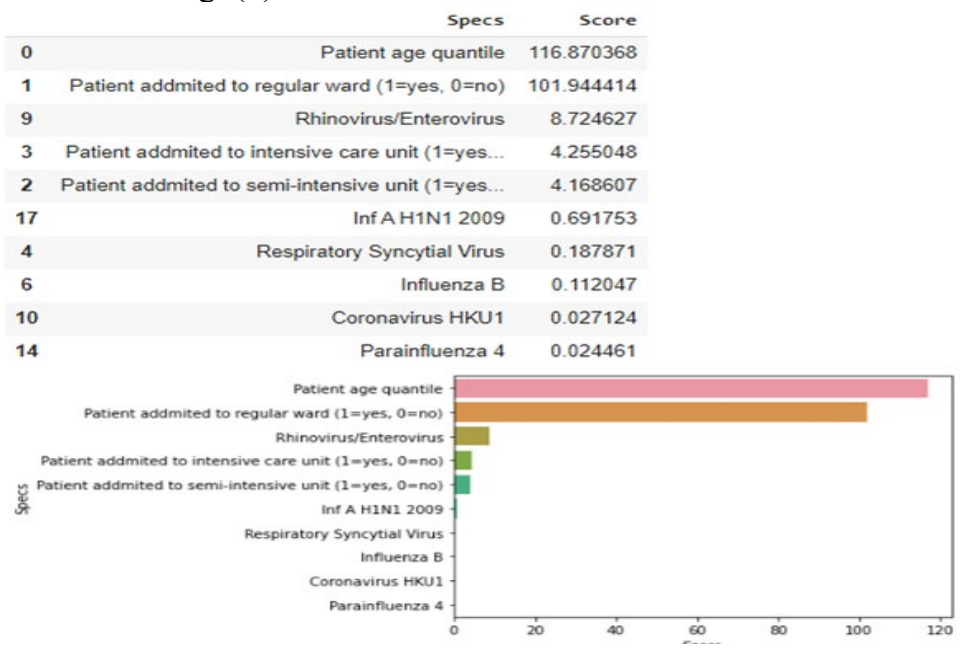

Fig. (3) Feature Importance - Efficient Random Forest Algorithm

Using the Model Characteristics property, the significance of each feature of theblood_reports Dataset is gained. To extract the top features from the dataset, the Extra Tree 
Classifier is used where feature importance is a built-in class with Tree Based classifiers. A score for every function is given by the Feature value and it determines the significance of the performance variable.

With the seaborn library, the correlation matrix with Heatmap is generated to indicate how the features are related to each other as well as the target variable. Fig. (4) shows the correlation values between the variables, which are positive or negative in nature.

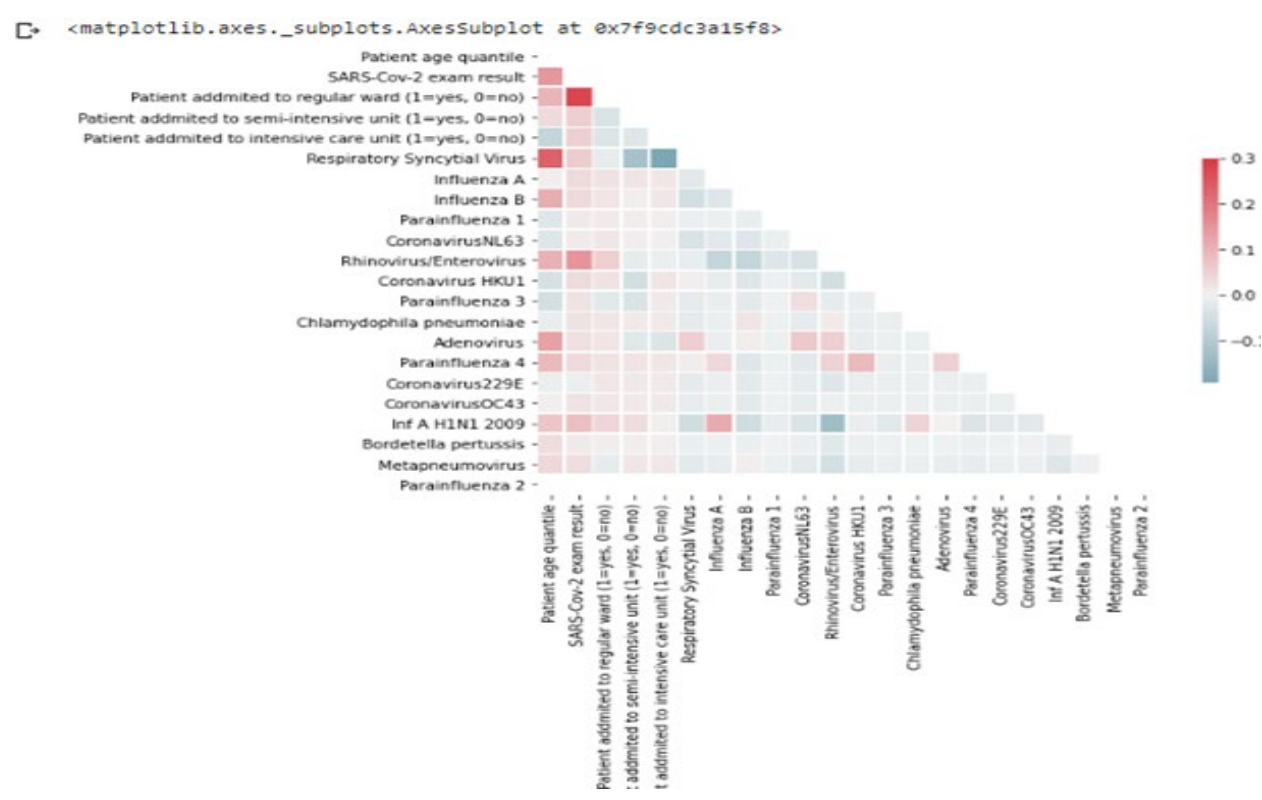

Fig. (4) Correlation Coefficient of attributes

\subsection{Experimental Analysis}

I. An Efficient Random Forest Algorithm:

Step- 1: Identified dataset from Kaggle.

Step- 2: Read the dataset using Pandas.

Import pandas as $\mathrm{p}$

data $=$ p.read_csv(“dataset.csv")

Step 3: Findout the missing values using

data.isnull().sum()

Step- 3: Drop non required columns likes serial number etc using data.drop(columnname)

Step- 4: Drop the columns which are having missing rate(null values of the data) $>20 \%$ using

data.drop()

step- 5: Find the datatype of the attributes ( We need to use integer for better prediction.) dat = data.select_dtypes(['data type name']) to display all available attributes of that data type.

Step- 6: Convert them to Integer data type using Label Encoder. 
le $=$ preprocessing.LabelEncoder ()

step- 7: Plot the heatmap to find the corelation between featureswith

cmap $=$ sns.diverging_palette $(220,10$, as_cmap $=$ True $)$

sns.heatmap (corr, mask $=$ mask, $\mathrm{cmap}=\mathrm{cmap}, \mathrm{vmax}=.3$,

center $=0$, square $=$ True, linewidths $=.5$, cbar_kws $=\{$ "shrink": .5 $\}$ )

Step- 7: Find out the importance of from the available features Using SelectKBest.

$\mathrm{X}=$ data.loc[:, data.columns != 'SARS-Cov-2 exam result']

$\mathrm{y}=$ data['SARS-Cov-2 exam result']

betfea $=$ SelectKBest $($ score func $=$ chi2, $\mathrm{k}=10$ )

Here we need to identify the predicting variable from our dataset and has to be stored in separate variable and then we need to perform the ChiSquareTest .

Plot a bar graph to understand the feature importance visually.

sns.barplot( $\mathrm{x}=$ "Score", $\mathrm{y}=$ "Specs", data $=$ featurescore)

Step- 8: Find the ratio of values present in predicting variable, it should be almost same else the prediction of the model is going to be biased. To avoid those scenarios we need to perform:

Find the ratio values present in predicting variable

If the ratio is almost same then

Skip this step

Else then

Perform Sampling.

To our dataset we are using RandomSampler.

rus $=$ RandomUnderSampler(random_state $=42$ ).

Step- 9: Split the datset into 2 parts among which one is used for training and other one will be used for testing.

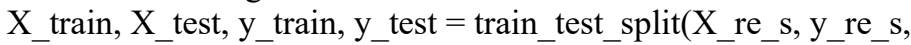

test_size $=0.1$, random_state $=42$ )

We splitted the data in the ratio of 90:10 in which

$90 \%$ iis used for training and $10 \%$ of data is usedfor testing.

Step- 10: Build and Train the model.

model_random $=$ RandomForestClassifier(criterion="gini",n_est

imators $=600$, min_samples_split $=5$, min_samples_leaf $=1$,

$\max$ features $=$ "auto",oob score $=$ True,random state $=1$ )

model_random.fit(X_train, y_train)

Step-11:Calculate and display the precision,recall and ROC:

Precision: scores.get('test_precision').mean())

Recall: scores.get('test_recall').mean())

ROC_ACU: scores.get('test_roc_auc').mean())

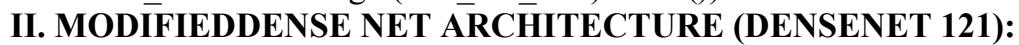

Step-1: First, we load the dataset then split it into covid and non covid (Using head and tail method)

Step-2: Then plot the count plot for finding the sampling.

Step-3: if(data is undersampled):

dodataagumentation process

else if (data is oversampled):

remove unnecessary data

else: 
go to next step

Step-4: Resize the given images to standard pixels(i.e 64)

Step-5.Resize the images using resize().

Step-6.Split the dataset into train and test in the ratio of 80:20 using test_train_split()

Step-7. Now, set values:

BATCH_SIZE $=64$

$\mathrm{EPOCHS}=25$

$\mathrm{SIZE}=64$

$\mathrm{N}$ chen $=3$

Step-8.Build the model() by using following inbuilt functions:

a. input()

b. convolution()

c. densnet()

d. GlobalAveragePooling2D()

e. BatchNormalization()

f. Dropout()

g. Dense()

h. BatchNormalization()

i. $\operatorname{dropout}()$

Step-9.stores the model using model variable

Step-10.optimize the model using adam optimizer

Step-11.run the model to findout the accuracy

Step-12. Testing the model

a.fit the model with test data using test dataset

b.Store the results

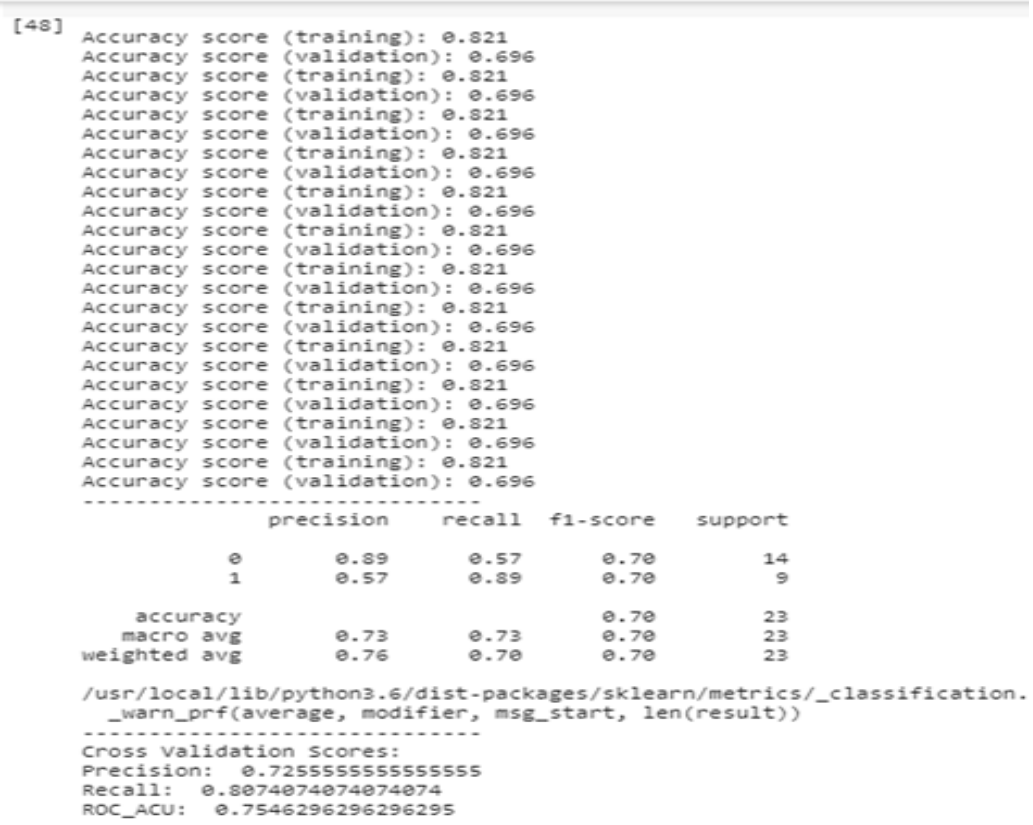

Fig. (5) Model Output 
4.4

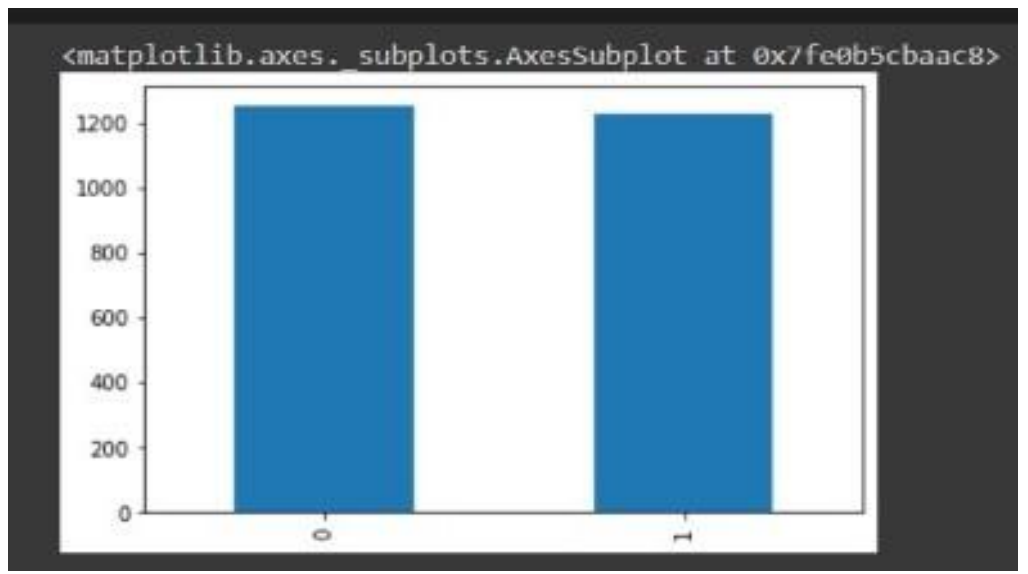

Simulation And Analysis:

Table 2.Comparison using $\mathrm{P}_{\mathrm{r}}$

Fig. (6) Samplified data

\begin{tabular}{|l|l|}
\hline Algorithm & Accuracy \\
\hline Gradient Boost Classification & $\mathbf{7 6 . 6 \%}$ \\
\hline Logistic Regression & $\mathbf{6 5 . 2 2 \%}$ \\
& \\
\hline Decision Tree Algorithm & $\mathbf{6 9 . 5 7 \%}$ \\
\hline Support Vector Machine & $\mathbf{7 9 . 8 2 \%}$ \\
\hline Random Forest Algorithm & $\mathbf{8 0 \%}$ \\
\hline An Efficient Random Forest Algorithm & $\mathbf{8 2 . 1 \%}$ \\
\hline
\end{tabular}

From the above table. 2 we concluded that Efficient Random Forest Algorithm is giving high accuracy than the other algorithms.An accuracy of $82.1 \%$ has been achieved after implementing Efficient Random Forest Algorithm on the blood_test_reports dataset.

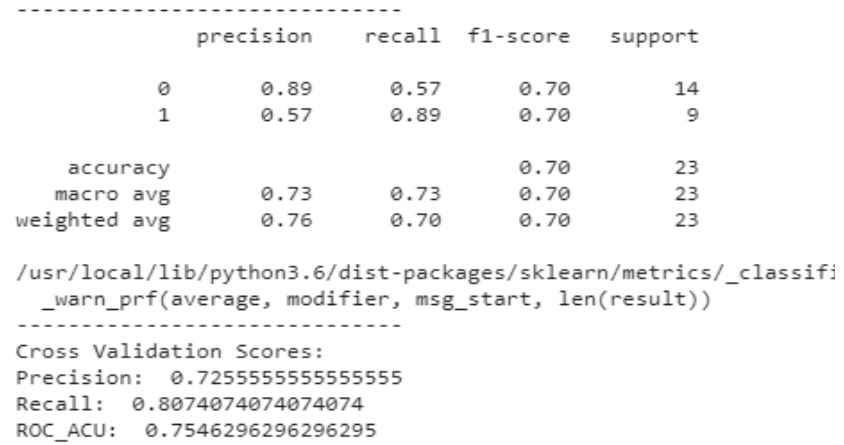

Fig. (7)Efficient Random Forest Confusion Matrix 
The Confusion Matrix shows the True Positive value and true Negative value.The ROC for Efficient Random Forest Algorithm is found and the Area under curve of the ROC is also found as best which says that the predictions are excellently made by the classifier.

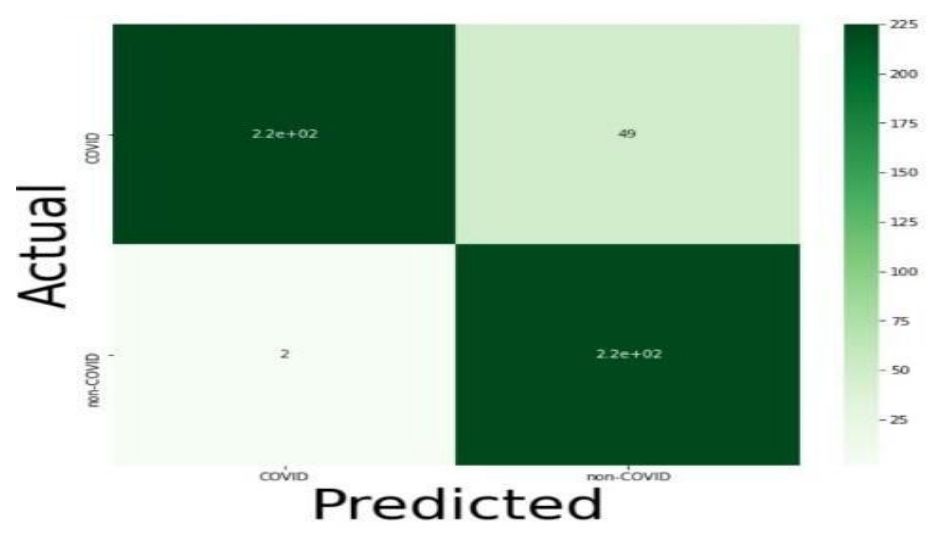

Fig. (8)Modified Densenet121 Confusion Matrix

The Confusion Matrix shows the True Positive value and true Negative values of the model.
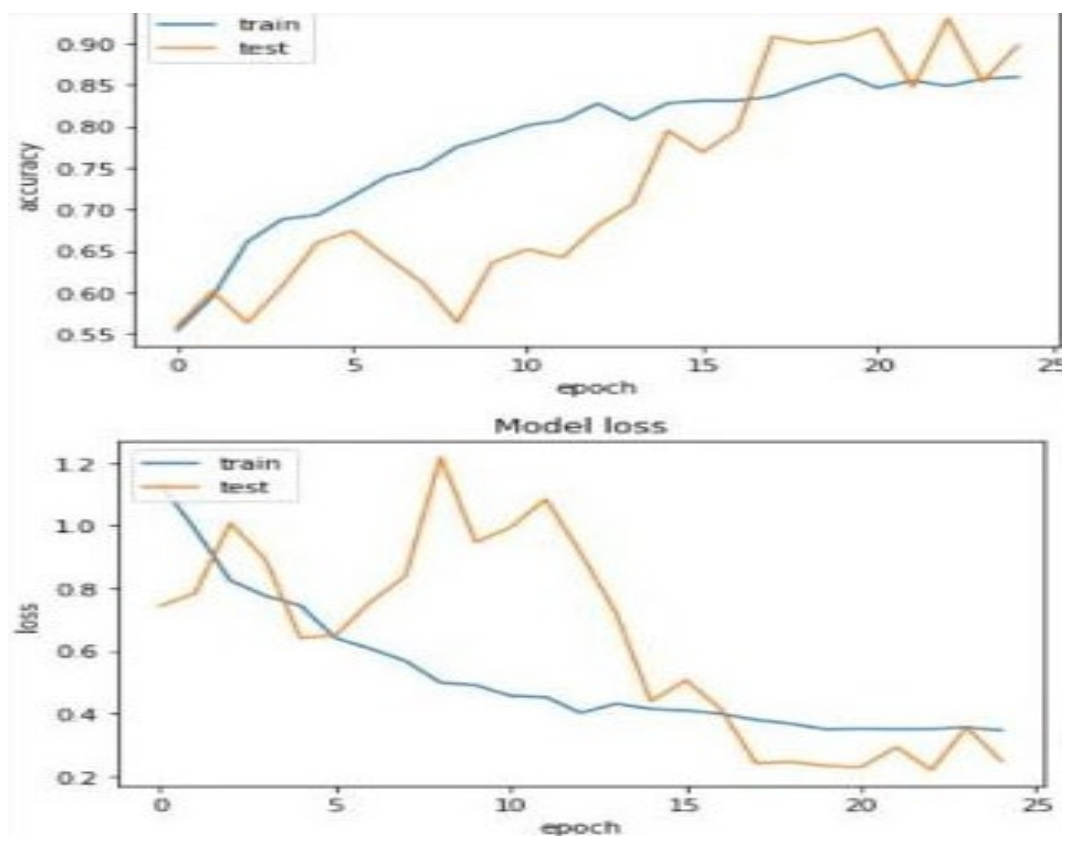

Fig. (9)ModifiedDensenet 121 Model Analysis 
The ROC for Densenet-121 Architecture Modelfound and the Area under curve of the $\mathrm{ROC}$ is also found as best which says that the predictions are excellently made by the classifier.

A web app has been deployed on the predictive model for the diagnosis of Covid patients. The web application takes the following features as input: Age, influenza, ,Adenovirus, parainfluenza etc. These are the most important features in predicting the risk of Covid-19 infection. After the input is gathered, the model runs and predicts whether the person is covid affected or not in an effective manner.

\section{Covid-19 predictions with Normal Blood Report}

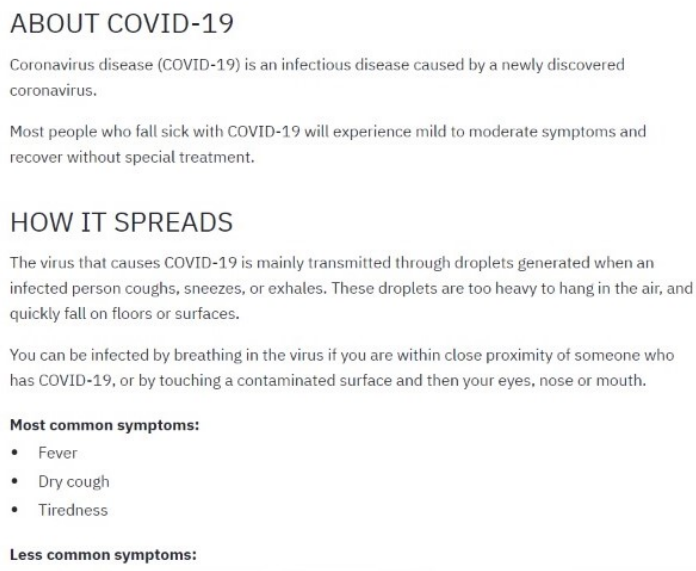

Fig. (10) Webapp Screenshot-1

Fill the folling form for COVID-19 TEST

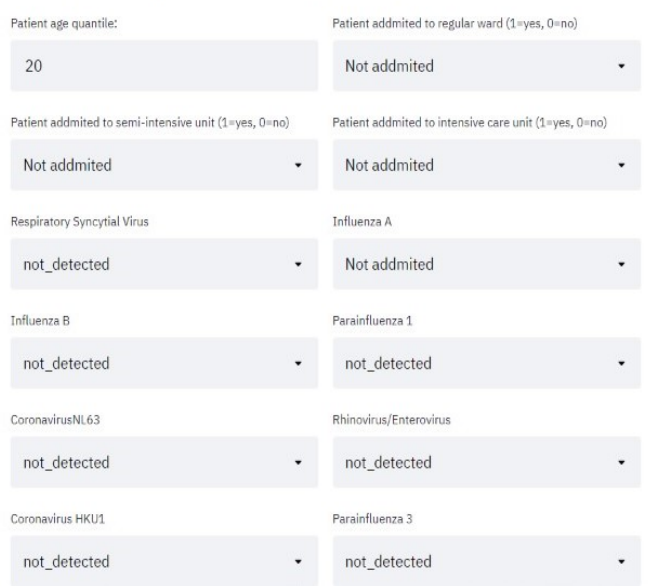

Fig. (11) Webapp screenshot-2 


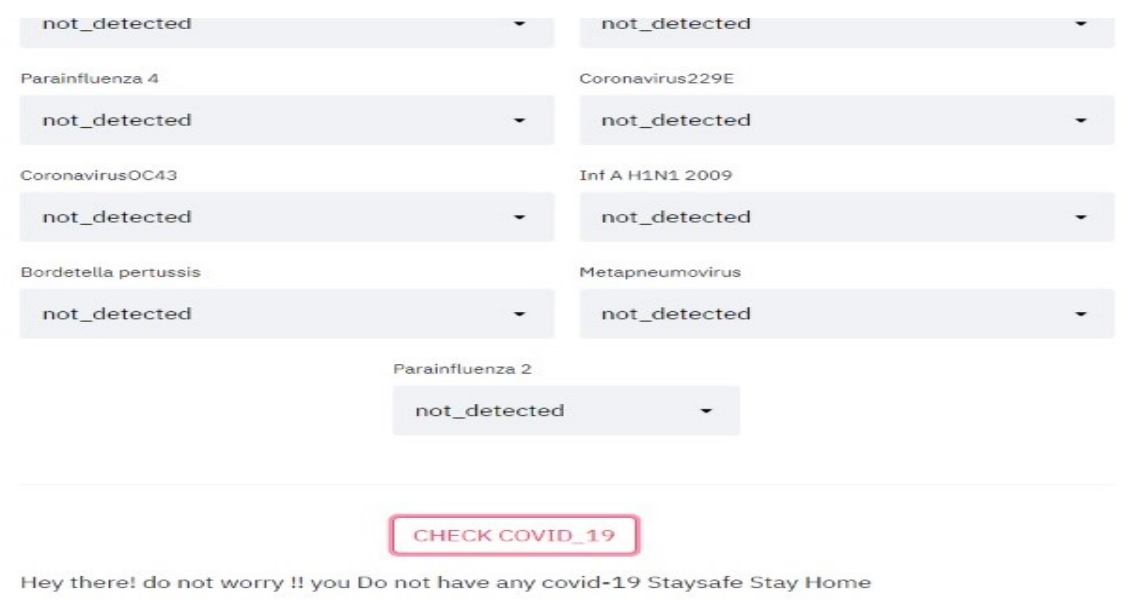

Fig. (12) Webapp screenshot-3

Upload File:

Limit $200 \mathrm{MB}$ per file • JPEG, PNG, JPG

Browse files

Please upload your file..........

Fig. (13) Webapp CT-Scan Screenshot

\section{Conclusion}

In the rapidly spreading of global pandemic there is a need to improve current testing methods by implementing Machine Learning in the medical field. Which will give fast and accurate results in short period of time. And also helps in early prediction of the virus. Our experimental outputs showing that the proposed methods(machine learning algorithm I.e..EfficientRandom Forest and deep learning algorithm I.e..ModifiedDensenet-121)was trained properly with large dataset.In future work we want to implement same procedure to predict covid-19 using CT Scan images. 


\section{ACKNOWLEDGEMENTS}

The final outcome and success of this project required a lot of assistance and guidance and we are extremely privileged to have this along the completion of the project. All that we have done is only due to such assistance and supervision and we thank our guides Dr. Meenakshi N and Dr. Kumaresan A for the support.

\section{References :}

[1] C. Wang, P. W. Horby, F. G. Hayden, and G. F. Gao, "A novel coronavirus outbreak of global health concern," Lancet, vol. 395, no. 10223, pp. 470-473, Feb. 2020.

[2] C. Huang et al., "Clinical features of patients infected with 2019 novel coronavirus in Wuhan, China," Lancet, vol. 395, no. 10223, pp. 497-506, Feb. 2020.

[3] F. Jiang, L. Deng, L. Zhang, Y. Cai, C. W. Cheung, and Z. Xia, "Review of the clinical characteristics of coronavirus disease 2019 (COVID-19)," Journal of General Internal Medicine, pp. 1-5, 2020.

[4] Z. Wu and J. M. McGoogan, "Characteristics of and important lessons from the coronavirus disease 2019 (COVID-19) outbreak in China: summary of a report of 72314 cases from the Chinese Center for Disease Control and Prevention," Jama, 2020.

[5] World Health Organization, "Laboratory testing for coronavirus disease 2019 (COVID-19) in suspected human cases: Interim guidance, 2 March 2020," World Health Organization, World Health Organization, 2020.

[6] T. Ai et al., "Correlation of chest CT and RT-PCR testing in coronavirus disease 2019 (COVID-19) in China: A report of 1014 cases,” Radiology, vol. 2019, Feb. 2020, Art. no. 200642.

[7] L. Li, L. Qin, Z. Xu, Y. Yin, X. Wang, B. Kong, J. Bai, Y. Lu, Z. Fang, Q. Song, K. Cao, D. Liu, G. Wang, Q. Xu, X. Fang, S. Zhang, J. Xia, and J. Xia, “Artificial intelligence distinguishes COVID-19 from community acquired pneumonia on chest CT,’' Radiology, vol. 296, Mar. 2020.

[8] S. Ahuja, B. Panigrahi, N. Dey, T. Gandhi, and V. Rajinikanth, "Deep transfer learning-based automated detection of COVID-19 from lung CT scan slices," Techrxiv, 2020

[9] M. Anthimopoulos, S. Christodoulidis, L. Ebner, A. Christe, and S. Mougiakakou, "Lung pattern classification for interstitial lung diseases using a deep convolutional neural network," IEEE Trans. Med. Imag., vol. 35, no. 5, pp. 1207-1216, May 2016. 
[10] Safavian SR, Landgrebe D. A survey of decision tree classifier methodology.IEEE transactions on systems, man, and cybernetics. 1991;21(3):660-674. doi: 10.1109/21.97458.

[11] Kam H.T.: Random decision forest.. In: Proceedings of the 3rd International Conference on Document Analysis and Recognition, Montreal, Canada, August, vol 1416, p 278282, 1995

[12] Schölkopf B, Smola A.J., Bach F., et al., 2002 Learning with kernels: support vector machines, regularization, optimization, and beyond. MIT press

[13] A. A. Ogunleye and W. Qing-Guo, "XGBoost model for chronic kidney disease diagnosis," IEEE/ACM transactions on computational biology and bioinformatics, 2019.

[14] M. Kim, J. Yun, Y. Cho, K. Shin, R. Jang, H.-J. Bae, and N. Kim, “'Deep learning in medical imaging," Neurospine, vol. 16, no. 4, pp. 657-668, 2019

[15] Y. LeCun, Y. Bengio, and G. Hinton,"Deep learning," nature, vol. 521, pp. 436-444, 2015

[16] C. Feng, A. Elazab, P. Yang, T. Wang, F. Zhou, H. Hu, et al., "Deep Learning Framework for Alzheimer's Disease Diagnosis via 3D-CNN and FSBi-LSTM," IEEE Access, vol. 7, pp. 63605-63618, 2019.

[17] C. Rachna. (2020, 15 April 2020). Difference betweenX-ray and CT Scan.

[18] F. Jiang, L. Deng, L. Zhang, Y. Cai, C. W. Cheung,and Z. Xia, "Review of the clinical characteristics of $\quad$ coronavirus disease 2019 (COVID-19)," Journal of General Internal Medicine, pp. 1-5, 2020.

[19] S. Wang, B. Kang, J. Ma, X. Zeng, M. Xiao, J. Guo, et al., "A deep learning algorithm using CT images to screen for Corona Virus Disease (COVID-19)," medRxiv, 2020.

[20] P. K. Sethy and S. K. Behera, "Detection of coronavirus Disease (COVID-19) based on Deep Features," 2020.

[21] Gino Sophia, Ceronmani Sharmila, \&quot;Recognition, Classification for Normal,Contact and Cosmetic Iris Images using Deep Learning\&quot; International Journal of Engineering and Advanced Technology, Vol. No. 8, Issue No. 3, ISSN : 2277-3878, pp. 4334-4340, September 2019. 